\title{
From Euler Diagrams in Schopenhauer to Aristotelian Diagrams in Logical Geometry
}

\author{
Lorenz Demey
}

\begin{abstract}
In this paper I explore the connection between Schopenhauer's Euler diagrams and the Aristotelian diagrams that are studied in contemporary logical geometry. One can define the Aristotelian relations in a very general fashion (relative to arbitrary Boolean algebras), which allows us to define not only Aristotelian diagrams for statements, but also for sets. I show that, once this generalization has been made, each of Schopenhauer's concrete Euler diagrams can be transformed into a well-defined Aristotelian diagram. More importantly, I also argue that Schopenhauer had several more general, systematic insights about Euler diagrams, which anticipate general insights and theorems about Aristotelian diagrams in logical geometry. Typical examples include the correspondence between $n$-partitions and $\alpha$-structures (a particular class of Aristotelian diagrams), and the fact that many families of Aristotelian diagrams have distinct Boolean subtypes. Because of his various concrete Euler diagrams and, especially, his more systematic observations about them, Schopenhauer can rightly be considered a distant forerunner of contemporary logical geometry.
\end{abstract}

Mathematics Subject Classification (2000). Primary 03A05; Secondary 03G05 68T30, 01A55.

Keywords. Euler diagram, Aristotelian diagram, Schopenhauer, logical geometry, square of opposition, $\alpha$-structure, bitstring.

\section{Introduction}

Arthur Schopenhauer (1788-1860) is best known for his deeply pessimistic outlook on the world, a world that he took to be cruel and filled with violence. By emphasizing the fundamental irrationality and absurdity of the universe, he not only anticipated much of 20th-century continental philosophy, but also influenced major literary figures such as Samuel Beckett and Jorge Luis Borges [35]. Much less known is that Schopenhauer was also concerned with what is perhaps the 
purest of all rational undertakings, viz. logic. There is a relatively brief discussion of logic in the first of the four books of his magnum opus, The World as Will and Representation [29], and a much more extensive treatment in a set of lecture notes [28], which were meant for his university lectures in Berlin in the 1820s [21, pp. 113-115].

Unsurprisingly, Schopenhauer holds logic in rather low esteem in everyday use, since he deems the discipline to be utterly useless for the purposes of daily life:

We no more need logic to avoid false reasoning than we need its rules to help us reason correctly; and even the most learned logician completely puts it aside when actually thinking. [29, p. 68]

Nevertheless, he does recognize the special epistemological status of logic, and therefore concludes that it is worth studying after all:

Even though it has no practical use, logic must nevertheless be preserved because of its philosophical interest as a special branch of knowledge concerning the organization and action of reason $[\ldots]$ it is a self-contained, self-subsistent, internally complete and perfect discipline that achieves absolute certainty. [29, pp. 69-70]

This double perspective on the discipline of logic (practically useless, but philosophically valuable) is also manifested in his remarks on the origin and history of logic [29, pp. 70-72].

In his discussion on logic, Schopenhauer makes extensive use of so-called Euler diagrams to visually represent the relationship between concepts. He also briefly touches upon the historical development of these diagrams: ${ }^{1}$

every concept has what may be termed an extension [Umfang] or sphere, even in cases where only a single real object corresponds to it [...] The idea of presenting these spheres by means of spatial figures is very felicitous. It occurred first to Gottfried Ploucquet, who used squares to do it; Lambert, who came after him, used plain lines positioned under each other; but it was Euler who perfected the idea by using circles. [29, p. 65]; also cf. [28, pp. $286-$ $287]$

Schopenhauer takes Euler diagrams to be absolutely central to logic. They not only facilitate the teaching of this discipline, but also capture its very essence, by allowing one to derive all the logical inference rules (also cf. [21, pp. 115-118]):

This schematism of concepts, which is already explained quite well in many textbooks, can be used to ground the doctrine of judgement as well as the whole of syllogistic logic and makes it very easy and uncomplicated to teach them both. [...] The essence of thought proper, i.e. of judgement and inference, can be presented

\footnotetext{
${ }^{1}$ More detailed (and historically accurate) accounts of the history of Euler-type diagrams can be found in $[2,3,21,22,23]$.
} 
by combining conceptual spheres according to the spatial schema described above, and all the rules of judgement and inference can be derived from this schema by construction. [29, pp. 68-70]

In this paper, I will explore the connection between Schopenhauer's Euler diagrams and another type of logical diagrams, viz. Aristotelian diagrams. Such diagrams visualize the Aristotelian relations of contradiction, contrariety, etc. that hold between a given number of propositions. The oldest and most well-known example of an Aristotelian diagram is the so-called 'square of opposition' for the categorical statements from syllogistics, but there are also many other (larger, more complex) Aristotelian diagrams, often developed in very different logical systems than traditional syllogistics. In contemporary logic, it has become clear that these diagrams can be fruitfully studied as objects of independent mathematical and philosophical interest, which has led to the burgeoning subfield of logical geometry $[6,8,11,16,32,33]$.

As far as I know, no square of opposition (or any other Aristotelian diagram) drawn by Schopenhauer has been handed down to us. ${ }^{2}$ Nevertheless, I will show in this paper that the particular Euler diagrams used by Schopenhauer can be 'translated' or 'transformed' into particular Aristotelian diagrams. More importantly, I will also argue that Schopenhauer formulated some more general insights about entire 'classes' or 'series' of Euler diagrams, which again have direct analogues in the realm of Aristotelian diagrams. Because of these systematic observations regarding entire classes of logic diagrams, Schopenhauer can rightly be considered a distant forerunner of contemporary logical geometry.

Before continuing, I should briefly say something about the scope of this paper. My argumentation will primarily be based on the relatively small number of Euler diagrams that appear in The World as Will and Representation [29]. There is also a plethora of Euler diagrams in Schopenhauer's university lecture notes [28], but for reasons of space, those diagrams will not be the main focus of this paper. I will only draw upon material from the lecture notes insofar as it allows me to further illustrate or reinforce a key claim in my overall argumentation. Finally, I will not say anything in this paper about the very large and complex 'Bonum/Malum diagram' [29, p. 74]. Schopenhauer himself already indicates that this diagram does not belong to logic proper, but rather to "the art of persuasion [Überredungskunst]" [29, p. 72]. A more detailed discussion of this particular diagram can be found in [24].

\footnotetext{
${ }^{2}$ In his university lecture notes, Schopenhauer did explicitly discuss the opposition relations that hold between the categorical statements [28, pp. 305ff.]. Furthermore, in the immediate textual surroundings of this discussion (e.g. on p. 297 and p. 304), we frequently find the reference "Illustr.", which indicates that an actual diagram is missing from the manuscript, probably because Schopenhauer drew it from memory during his actual lectures. It can reasonably be assumed that at least one of these occurrences of "Illustr." refers to a square of opposition. Thanks to Jens Lemanski for some interesting discussion about this.
} 
The paper is organized as follows. Section 2 provides some necessary background on logical geometry, focusing on a very general way of defining the Aristotelian relations, and a particular class of Aristotelian diagrams, viz. the so-called $\alpha$-structures. Section 3 shows how two of Schopenhauer's simplest Euler diagrams can be transformed into well-defined Aristotelian diagrams, viz. a classical and a degenerate square of opposition. Section 4 deals with Schopenhauer's infinite series of Euler diagrams for partitions, and shows how it can be transformed into an infinite series of strong $\alpha$-structures. Section 5 is concerned with a final class of Euler diagrams, and shows how each of them can be transformed into both a strong and a weak $\alpha$-structure. Section 6 briefly summarizes the results that have been obtained in this paper.

\section{Some Background on Logical Geometry}

This section provides some necessary background on logical geometry. In Subsection 2.1, I define the Aristotelian relations with respect to an arbitrary Boolean algebra. This will later enable us to transform Schopenhauer's Euler diagrams into well-defined Aristotelian diagrams. Next, in Subsection 2.2, I introduce a particular class of Aristotelian diagrams, viz. the so-called $\alpha$-structures, and discuss some of their key properties. These $\alpha$-structures will turn out to play a crucial role in Sections 4 and 5 .

\subsection{Defining the Aristotelian Relations in a Boolean Algebra}

The Aristotelian relations can be characterized with various degrees of abstractness and generality $[10,12]$. For the purposes of this paper, it will be useful to consider a very general definition, in the mathematical setting of Boolean algebra [17]; afterwards, I will show how the more well-known characterizations of the Aristotelian relations can be obtained as special cases of this definition.

Definition 2.1. Let $\mathbb{B}=\langle B, \wedge, \vee, \neg, \top, \perp\rangle$ be an arbitrary Boolean algebra. Two elements $x, y \in B$ are said to be

$$
\begin{aligned}
& \mathbb{B} \text {-contradictory iff } \quad x \wedge y=\perp \quad \text { and } \quad x \vee y=\top \text {, } \\
& \mathbb{B} \text {-contrary iff } \quad x \wedge y=\perp \quad \text { and } \quad x \vee y \neq \top \text {, } \\
& \mathbb{B} \text {-subcontrary iff } \quad x \wedge y \neq \perp \quad \text { and } \quad x \vee y=\top \text {, } \\
& \text { in } \mathbb{B} \text {-subalternation iff } \neg x \vee y=\top \quad \text { and } \quad x \vee \neg y \neq \top \text {. }
\end{aligned}
$$

Note that by De Morgan's laws, the condition $x \vee y=\top$ is equivalent to $\neg x \wedge \neg y=$ $\perp$, while the conditions $\neg x \vee y=\top$ and $x \vee \neg y \neq \top$ are equivalent to resp. $x \wedge \neg y=$ $\perp$ and $\neg x \wedge y \neq \perp$. This means that the relations of contradiction, contrariety and subcontrariety are all defined in terms of the 'symmetrical' elements $x \wedge y$ and $\neg x \wedge \neg y$, whereas the relation of subalternation is defined in terms of the 'asymmetrical' elements $\neg x \wedge y$ and $x \wedge \neg y$. This conceptual split in the definitions of the Aristotelian relations is explored in much more detail in [31].

Definition 2.1 provides a characterization of the Aristotelian relations in an arbitrary Boolean algebra. However, it is also important to have a clear grasp of 
what it means for two elements not to stand in any Aristotelian relation whatsoever. This corresponds to the notion of unconnectedness $[15,31]$, which is defined in terms of four conditions:

Definition 2.2. Let $\mathbb{B}=\langle B, \wedge, \vee, \neg, \top, \perp\rangle$ again be an arbitrary Boolean algebra. Two elements $x, y \in B$ are said to be $\mathbb{B}$-unconnected iff (i) $x \wedge y \neq \perp$ and (ii) $x \wedge$ $\neg y \neq \perp$ and (iii) $\neg x \wedge y \neq \perp$ and (iv) $\neg x \wedge \neg y \neq \perp$.

The first condition of Definition 2.2 implies that $x$ and $y$ are neither $\mathbb{B}$-contradictory nor $\mathbb{B}$-contrary, while the fourth condition implies that $x$ and $y$ are neither $\mathbb{B}$ contradictory nor $\mathbb{B}$-subcontrary. The second condition implies that there is no $\mathbb{B}$-subalternation from $x$ to $y$, and similarly, the third condition implies that there is no $\mathbb{B}$-subalternation from $y$ to $x$. Together, these four conditions thus imply that the elements $x, y \in B$ do not stand in any Aristotelian relation in the Boolean algebra $\mathbb{B}$.

We can move from these very general definitions to more well-known characterizations of the Aristotelian relations and unconnectedness, by plugging in a concrete Boolean algebra for $\mathbb{B}$. I will now discuss two key examples: (i) letting $\mathbb{B}$ be a Boolean algebra of statements, and (ii) letting $\mathbb{B}$ be a Boolean algebra of sets. $^{3}$

First of all, consider the case where $\mathbb{B}$ is a Boolean algebra of statements. (This can typically be achieved by taking $\mathbb{B}$ to be the Lindenbaum-Tarski algebra of some suitable logical system S. ${ }^{4}$ ) The top and bottom elements of such a Boolean algebra are resp. the tautological and self-contradictory statements, while the algebraic operations of meet, join and complement correspond to the logical operations of resp. conjunction, disjunction and negation. By applying Definition 2.1, we find that two statements $P$ and $Q$ are contrary in this Boolean algebra iff $P \wedge Q=\perp$ and $P \vee Q \neq \top$, i.e. iff the conjunction of $P$ and $Q$ is self-contradictory, while the disjunction of $P$ and $Q$ is not tautological. The first part means exactly that $P$ and $Q$ cannot be true together, while the second part means that $P$ and $Q$ can be false together. We have thus obtained the 'familiar' definition of contrariety for statements (in terms of being able to be true/false together) as a special case of Definition 2.1. The familiar definitions of contradiction, subcontrariety, subalternation and unconnectedness for statements can be obtained from Definitions 2.1 and 2.2 in a completely analogous fashion.

Secondly, consider the case where $\mathbb{B}$ is a Boolean algebra of sets. (Because of the Stone representation theorem, every Boolean algebra is isomorphic to a

\footnotetext{
${ }^{3}$ The close relationship between these two examples was already noted by Keynes, who wrote: "These seven possible relations between propositions (taken in pairs) will be found to be precisely analogous to the seven possible relations between classes (taken in pairs)" [19, p. 119, my emphases]. Note that Keynes talks about seven relations, because in addition to the four Aristotelian relations and unconnectedness, he is considering two others. However, this difference is irrelevant for our current purposes.

"Suitable' here means that the logical system $\mathrm{S}$ has all the connectives and axioms that are needed to guarantee that its Lindenbaum-Tarski algebra effectively is a Boolean algebra. This is mainly a technical caveat, and it is further irrelevant for the purposes of this paper.
} 
Boolean algebra of this kind [17].) The top and bottom elements of such a Boolean algebra are resp. some domain of discourse $D$ and the empty set $\emptyset$, while the algebraic operations of meet, join and complement correspond to the set-theoretical operations of resp. intersection, union and complementation (with respect to $D$ ). By applying Definition 2.1, we find that two sets $X$ and $Y$ are contrary in this Boolean algebra iff $X \cap Y=\emptyset$ and $X \cup Y \neq D$, i.e. iff the intersection of $X$ and $Y$ is empty, while the union of $X$ and $Y$ does not exhaust the domain of discourse $D$. The definitions of contradiction, subcontrariety, subalternation and unconnectedness for sets can be obtained from Definitions 2.1 and 2.2 in a completely analogous fashion. For future reference, all these definitions are listed below:

Definition 2.3. Let $\mathbb{B}=\langle B, \cap, \cup, \backslash, D, \emptyset\rangle$ be a Boolean algebra of sets. Two sets $X, Y \in B$ are said to be

$\begin{array}{lllll}\mathbb{B} \text {-contradictory } & \text { iff } & X \cap Y=\emptyset & \text { and } & X \cup Y=D, \\ \mathbb{B} \text {-contrary } & \text { iff } & X \cap Y=\emptyset & \text { and } & X \cup Y \neq D, \\ \mathbb{B} \text {-subcontrary } & \text { iff } & X \cap Y \neq \emptyset & \text { and } & X \cup Y=D, \\ \text { in } \mathbb{B} \text {-subalternation } & \text { iff } & (D \backslash X) \cup Y=D & \text { and } & X \cup(D \backslash Y) \neq D, \\ \mathbb{B} \text {-unconnected } & \text { iff } & X \cap Y \neq \emptyset & \text { and } & X \cap(D \backslash Y) \neq \emptyset \\ & \text { and } & (D \backslash X) \cap Y \neq \emptyset & \text { and } & (D \backslash X) \cap(D \backslash Y) \neq \emptyset .\end{array}$

Note that the conditions $(D \backslash X) \cup Y=D$ and $X \cup(D \backslash Y) \neq D$ are equivalent to resp. $X \subseteq Y$ and $X \nsupseteq Y$. In a Boolean algebra of sets, subalternation thus corresponds to the proper subset-relation: there is a subalternation from $X$ to $Y$ iff $X \subset Y$.

Because of the generality of Definitions 2.1 and 2.2, we are now able to deal with Aristotelian relations not only in the case of statements, but also in the case of sets. This insight will be absolutely crucial when we transform Schopenhauer's Euler diagrams into Aristotelian diagrams, because those Euler diagrams also represent relations between sets. More specifically, Schopenhauer's Euler diagrams represent relations between spheres/extensions of concepts (cf. the quotation provided in Section 1), and these extensions are indeed sets. For example, the extension of the concept Horse is the set of all concrete horses that exist in the world.

\subsection{The $\alpha$-Structures and their Properties}

Now that the Aristotelian relations and unconnectedness have been defined relative to arbitrary Boolean algebras, we can likewise define the notion of an Aristotelian diagram:

Definition 2.4. Let $\mathbb{B}=\langle B, \wedge, \vee, \neg, \top, \perp\rangle$ be an arbitrary Boolean algebra and consider a fragment $\mathcal{F} \subseteq B \backslash\{\top, \perp\}$. Suppose that $\mathcal{F}$ is closed under $\mathbb{B}$-complementation, i.e. if $x \in \mathcal{F}$ then $\neg x \in \mathcal{F}$. An Aristotelian diagram for $\mathcal{F}$ in $\mathbb{B}$ is a diagram that visualizes an edge-labeled graph $\mathcal{G}$. The vertices of $\mathcal{G}$ are the elements of $\mathcal{F}$, and the edges of $\mathcal{G}$ are labeled by the Aristotelian relations holding in $\mathbb{B}$ between those elements, i.e. if $x, y \in \mathcal{F}$ stand in some Aristotelian relation in $\mathbb{B}$, then this is visualized according to the code in Fig. 1(a). 
Note that by definition, the set $\mathcal{F}$ of elements appearing in an Aristotelian diagram is closed under complementation and only contains non-trivial elements (i.e. neither $\top$ nor $\perp$ ). There are various historical and systematic reasons for these restrictions [31, Subsection 2.1], which need not concern us here. Later on in this paper, we will focus on a particular subclass of Aristotelian diagrams, viz. the so-called $\alpha$-structures or $\alpha$-diagrams (the term ' $\alpha$-structure' is due to Moretti [25]): ${ }^{5}$

Definition 2.5. Let $\mathbb{B}$ again be a Boolean algebra, and let $n \geq 1$ be a natural number. An $\alpha_{n}$-structure in $\mathbb{B}$ is an edge-labeled graph $\mathcal{G}$. The vertices of $\mathcal{G}$ form a fragment $\left\{x_{1}, \ldots, x_{n}, \neg x_{1}, \ldots, \neg x_{n}\right\} \subseteq B \backslash\{\top, \perp\}$, where all distinct $x_{i}, x_{j}$ are pairwise $\mathbb{B}$-contrary, i.e. such that $x_{i}$ and $x_{j}$ are $\mathbb{B}$-contrary for all $1 \leq i \neq j \leq n$. The edges of $\mathcal{G}$ are labeled by the Aristotelian relations holding in $\mathbb{B}$ between those elements An $\alpha_{n}$-diagram in $\mathbb{B}$ is an Aristotelian diagram that visualizes such an $\alpha_{n}$-structure in $\mathbb{B}$.

The condition of pairwise $\mathbb{B}$-contrariety between all distinct $x_{i}, x_{j}$ immediately implies that there are several other Aristotelian relations in an $\alpha_{n}$-structure as well. In particular, it follows that $\neg x_{i}$ and $\neg x_{j}$ are $\mathbb{B}$-subcontrary and that there are $\mathbb{B}$-subalternations from $x_{i}$ to $\neg x_{j}$, for all $1 \leq i \neq j \leq n$. Furthermore, as in any Aristotelian diagram, it holds that $x_{i}$ and $\neg x_{i}$ are $\mathbb{B}$-contradictory, for all $1 \leq i \leq n$.

Although there certainly exist Aristotelian diagrams that do not belong to the class of $\alpha$-structures, this particular class does contain some of the most wellknown examples of Aristotelian diagrams. For example, consider the four smallest members of this class, i.e. the $\alpha_{n}$-structures for $n \in\{1,2,3,4\}$ :

- The $\alpha_{1}$-structure is simply a pair of contradictory elements (PCD). ${ }^{6}$ An example is shown in Fig. 1(b). Because Aristotelian diagrams are supposed to be closed under complementation, this is the smallest possible Aristotelian diagram. PCDs do not frequently appear in the literature, but they have considerable theoretical importance, since they can be thought of as the fundamental 'building blocks' for all other, larger Aristotelian diagrams [13, 14].

- The $\alpha_{2}$-structure is a classical square of opposition. An example is shown in Fig. 1(c). Without a doubt, this is the oldest, most well-known and most frequently-used type of Aristotelian diagram.

- The $\alpha_{3}$-structure is a so-called Jacoby-Sesmat-Blanché hexagon (JSB hexagon), which is named after Jacoby [18], Sesmat [30] and Blanché [5]. An example is shown in Fig. 1(d). After the classical square of opposition, this is probably

\footnotetext{
${ }^{5}$ Strictly speaking, the term ' $\alpha$-structure' refers to the (abstract) underlying graph structure, while the term ' $\alpha$-diagram' refers to the (concrete) diagrammatic visualization. However, for the purposes of this paper, this distinction will not matter much, so we will usually not distinguish between these two terms, and follow Moretti [25] in simply talking about ' $\alpha$-structures'.

${ }^{6}$ Note that an $\alpha_{1}$-structure does not contain any distinct $x_{i}, x_{j}$, and hence, no contrarieties either. Since the pairwise contrarieties among distinct $x_{i}, x_{j}$ constitute the characteristic feature of the $\alpha$-structures (cf. Definition 2.5), the $\alpha_{1}$-structure is clearly seen to be a 'limiting' (or 'degenerate') case of the $\alpha$-structures in general.
} 
Figure 1. (a) code for visually representing the Aristotelian relations; examples of (b) PCD, (c) classical square of opposition,

(d) JSB hexagon, (e) Moretti octagon.

(a)

\author{
contradiction \\ contrariety
}

(b)

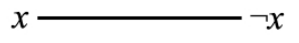

(c)

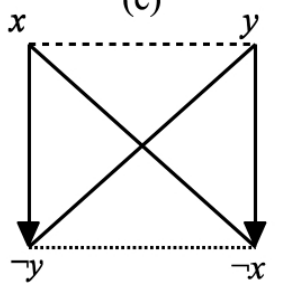

(d)

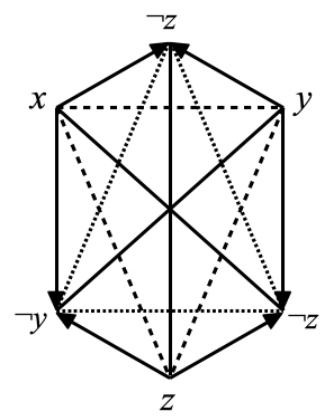

subcontrariety

subalternation

(e)

the most well-known and most frequently-used type of Aristotelian diagram. Within a JSB hexagon, one can discern a triangle of contraries that interlocks with a triangle of subcontraries.

- The $\alpha_{4}$-structure is a so-called Moretti octagon, which is named after Moretti [25] (who drew it as a cube, rather than an octagon). An example is shown in Fig. 1(e). Within a Moretti octagon, one can discern a trapezoid of contraries and a trapezoid of subcontraries. (If this diagram is drawn as a cube instead of an octagon, then these two trapezoids correspond to a tetrahedron of contraries that interlocks with a tetrahedron of subcontraries. Such a tetrahedron of contraries was already known (and drawn) by Charles S. Peirce; cf. $[1$, p. 60] and [20, p. 569].)

One of the main theoretical insights of logical geometry is that a given family of Aristotelian diagrams can have multiple Boolean subtypes, i.e. it is perfectly possible for two Aristotelian diagrams to exhibit exactly the same configuration of Aristotelian relations among their respective sets of elements, and yet have completely different Boolean properties $[9,15]$. The first concrete example of this phenomenon was pointed out by Pellissier [26], and concerns the JSB hexagons. One can show that there are two Boolean subtypes of JSB hexagons, with completely different Boolean properties: in a strong JSB hexagon, the join of the 3 contrary elements equals T, whereas in a weak JSB hexagon, the join of the 3 contrary elements is not equal to $T$. These kinds of (differences in) Boolean properties are nowadays usually characterized in terms of bitstring length, i.e. the smallest 
number of bits with which a given diagram can be encoded. For example, a strong JSB hexagon can be encoded by bitstrings of length 3 (its 3 contrary elements are then encoded as 100, 010 and 001 , so that $100 \vee 010 \vee 001=111$ ), whereas a weak JSB hexagon requires bitstrings of length 4 (its 3 contrary elements are then encoded as 1000,0100 and 0010 , so that $1000 \vee 0100 \vee 0010=1110 \neq 1111$ ) $[15,34] .^{7}$

We are now in a position to systematically examine the Boolean subtypes of the various $\alpha$-structures. Theorem 2.6 below summarizes the situation. For reasons of space, this theorem will not be proved in this paper, but it is based on a straightforward application of bitstring analysis. Note that the important cutoff happens at $n=3$. This is not a coincidence: because of their binary nature, the Aristotelian relations cannot capture the full Boolean complexity that may arise in larger sets [7]. ${ }^{8}$

\section{Theorem 2.6.}

1. The family of $\alpha_{1}$-structures is Boolean homogeneous, i.e. it has just a single Boolean subtype, which requires bitstrings of length 2.

2. The family of $\alpha_{2}$-structures is Boolean homogeneous, i.e. it has just a single Boolean subtype, which requires bitstrings of length 3.

3. For $n \geq 3$, the family of $\alpha_{n}$-structures has two Boolean subtypes: (i) a strong subtype, which requires bitstrings of length $n$, and (ii) a weak subtype, which requires bitstrings of length $n+1$.

The cases $n=1$ and $n=2$ of this theorem mean that the family of PCDs and the family of classical squares of opposition are both Boolean homogeneous, which is well-known in logical geometry. The case $n=3$ means that the family of JSB hexagons has two Boolean subtypes, viz. the strong JSB hexagons (which require bitstrings of length 3) and the weak JSB hexagons (which require bitstrings of length 4). We have already seen that this was first pointed out by Pellissier [26]. For a final example, note that the case $n=4$ means that the family of Moretti octagons has two Boolean subtypes, viz. the strong Moretti octagons (which require bitstrings of length 4) and the weak Moretti octagons (which require bitstrings of length 5). A concrete example of a strong Moretti octagon can be found in Moretti [25] (drawn as a cube), while a weak Moretti octagon can be found in Reichenbach [27] (again drawn as a cube).

In a Boolean algebra $\mathbb{B}=\langle B, \wedge, \vee, \neg, \top, \perp\rangle$, a finite set $\Pi=\left\{x_{1}, \ldots, x_{n}\right\} \subseteq$ $B \backslash\{\top, \perp\}$ (with $n \geq 2$ ) is said to be an $n$-partition of $\mathbb{B}$ iff (i) $x_{i} \wedge x_{j}=\perp$ for all distinct $x_{i}, x_{j} \in \Pi$ and (ii) $\bigvee \Pi=\top$. There is a clear correspondence between partitions and (strong) $\alpha$-structures. This is made fully precise in Theorem 2.7 below.

\footnotetext{
${ }^{7}$ There also exist Aristotelian families that have more than two Boolean subtypes. For example, the family of Buridan octagons has three Boolean subtypes: one that requires bitstrings of length 4 , one that requires bitstrings of length 5 , and one that requires bitstrings of length 6 [9, 15]. ${ }^{8}$ For an easy illustration from classical propositional logic, note that the 3-element set $\{p \vee$ $q, \neg p, \neg q\}$ is inconsistent, while each of its 2-element subsets is consistent.
} 
Note that there is again a cutoff at $n=3$, and that $\alpha_{2}$-structures (i.e. classical squares of opposition) do not correspond to any partitions.

\section{Theorem 2.7.}

1. Each 2-partition $\{x, \neg x\}$ gives rise to an $\alpha_{1}$-structure with elements $\{x, \neg x\}$, and a contradiction holding between $x$ and $\neg x$.

2. For $n \geq 3$, each 3-partition $\left\{x_{1}, \ldots, x_{n}\right\}$ gives rise to a strong $\alpha_{n}$-structure with elements $\left\{x_{1}, \ldots, x_{n}, \neg x_{1}, \ldots, \neg x_{n}\right\}$, with contradictions between $x_{i}$ and $\neg x_{i}$, and contrarieties between $x_{i}$ and $x_{j}$, for all $1 \leq i \neq j \leq n$.

The case $n=2$ of this theorem means that each 2-partition corresponds to a PCD. The case $n=3$ means that each 3 -partition corresponds to a strong JSB hexagon, which has the 3 elements of the partition on its triangle of contraries. The case $n=4$ means that each 4-partition corresponds to a strong Moretti octagon, which has the 4 elements of the partition on its trapezoid of contraries.

\section{Two Basic Examples}

After this brief overview of logical geometry, we are now ready to turn to Schopenhauer. In this section I will show how two of Schopenhauer's simplest Euler diagrams can be transformed into well-defined Aristotelian diagrams. This will be a valuable exercise in itself, but it will also serve as a useful preparation for the more involved transformations of Euler diagrams into Aristotelian diagrams that will be discussed in Sections 4 and 5.

\subsection{From an Euler Diagram to a Classical Square of Opposition}

Schopenhauer begins his discussion of Euler diagrams by mentioning the most basic case, viz. that of two concepts that completely coincide with each other. (The example he gives involves the concepts of Ruminantia and Bisulca, i.e. ruminants and animals with cloven hoofs.) He does not explicitly provide an Euler diagram for this situation, stating that "Such cases may be presented using a single circle that signifies the one as much as the other" [29, p. 66].

Next, Schopenhauer turns to the case where "The sphere of one concept completely encloses the sphere of another." [29, p. 66]. The example he gives involves the concepts of Horse and Animal; the accompanying Euler diagram is shown in Fig. 2(a). This diagram clearly shows that (the extension/sphere of) Horse is a proper subset of (the extension/sphere of) Animal. ${ }^{9}$ We have already seen in Subsection 2.1 that this proper subset-relation essentially amounts to a subalternation

\footnotetext{
${ }^{9}$ One might object that Schopenhauer's words ("completely enclose") commit him to Horse being a subset of Animal, but not necessarily a proper subset. This objection is misguided, for the following two reasons. First of all, the Euler diagram used by Schopenhauer contains a significant amount of space for the part Animal $\backslash$ Horse, which is a clear visual suggestion that Horse is a proper subset of Animal. Secondly, if the inclusion were non-proper, then it would be possible that Horse and Animal are exactly the same concept, so that they should have been represented by just a single circle instead (cf. the first case discussed by Schopenhauer).
} 
Figure 2. (a) Schopenhauer's Euler diagram; (b) the corresponding Aristotelian diagram: a classical square of opposition.

(a)

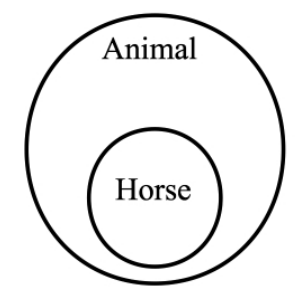

(b)

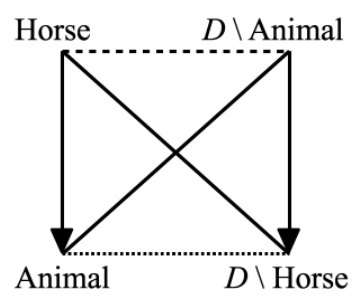

from Horse to Animal, in some underlying Boolean algebra of sets. The smallest Boolean algebra of sets that non-trivially ${ }^{10}$ contains Horse and Animal has $2^{3}=8$ elements. This Boolean algebra, $\mathbb{B}_{3}$, has top element $D$ (for Domain), bottom element $\emptyset$, and three atomic elements, viz. Horse, Animal $\backslash$ Horse and $D \backslash$ Animal. The Hasse diagram for $\mathbb{B}_{3}$ is shown in Fig. $3(\mathrm{a})$; note that $\mathbb{B}_{3}$ is isomorphic to the powerset algebra $\wp(\{1,2,3\})$.

We can now determine the Aristotelian relations holding between some of the sets of this Boolean algebra $\mathbb{B}_{3}$ (recall Definition 2.3). We have already seen that Horse $\subset$ Animal, which means exactly that there is a $\mathbb{B}_{3}$-subalternation from Horse to Animal. Furthermore, since Horse $\cap(D \backslash$ Horse $)=\emptyset$ and Horse $\cup(D \backslash$ Horse $)=D$, it follows that Horse and $D \backslash$ Horse are $\mathbb{B}_{3}$-contradictories; similarly, Animal and $D \backslash$ Animal are also $\mathbb{B}_{3}$-contradictories. Furthermore, since Horse $\cap(D \backslash$ Animal $)=\emptyset$ and Horse $\cup(D \backslash$ Animal $) \neq D$, it follows that Horse and $D \backslash$ Animal are $\mathbb{B}_{3^{-}}$ contraries. Completely analogously, one can show that Animal and $D \backslash$ Horse are $\mathbb{B}_{3}$-subcontraries, and that there is a $\mathbb{B}_{3}$-subalternation from $D \backslash$ Animal to $D \backslash$ Horse. All these Aristotelian relations can be summarized by means of a classical square of opposition, as shown in Fig. 2(b).

We have thus succeeded in transforming Schopenhauer's original Euler diagram (Fig. 2(a)) into an Aristotelian diagram, viz. a classical square of opposition (Fig. 2(b)). It bears emphasizing that the Aristotelian relations that are visualized by this square are all mathematically well-defined: they hold relative to the underlying Boolean algebra $\mathbb{B}_{3}$ (cf. Fig. 3(a)), and are thus specific instantiations of the general characterization of the Aristotelian relations provided by Definition 2.1. To finish this subsection, I will now make three remarks, in increasing order of importance.

Remark 3.1. The transformation process that has just been described is by no means an injection, i.e. it is perfectly possible for two distinct Euler diagrams to

\footnotetext{
${ }^{10}$ I.e. in such a way that neither Horse nor Animal end up being the top or bottom element of the Boolean algebra. See Remark 3.3 for a more detailed discussion.
} 
Figure 3. Hasse diagram for (a) the Boolean algebra $\mathbb{B}_{3}$ and (b) the Boolean algebra $\mathbb{B}_{2}$.

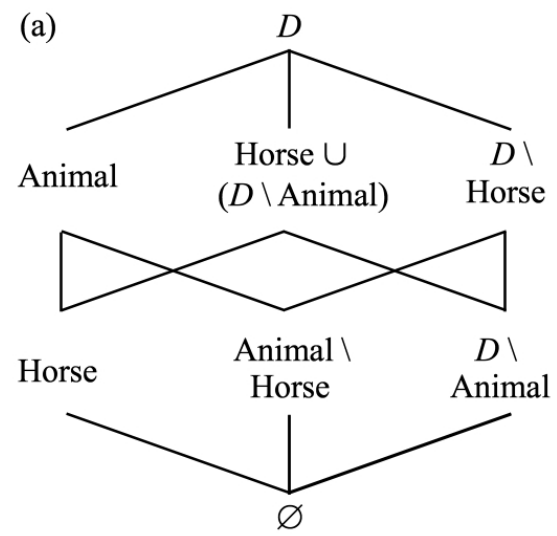

(b)

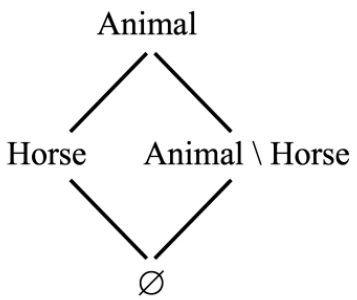

be transformed into one and the same Aristotelian diagram. For example, we have just seen how the Euler diagram for the proper inclusion of Horse in Animal is transformed into a classical square of opposition. It is easy to see how another Euler diagram, which visualizes the proper inclusion of $D \backslash$ Animal in $D \backslash$ Horse, would be transformed into exactly the same classical square. Alternatively, one can view the original Euler diagram in Fig. 2(a) as a visual representation of both proper inclusion relations - albeit, perhaps, with different degrees of visual perspicuity. More generally, from this alternative perspective, the single Euler diagram in Fig. 2(a) at once visualizes six relations among Horse, Animal, $D \backslash$ Horse and $D \backslash$ Animal, all six of which are also visualized by the classical square of opposition in Fig. 2(b).

Remark 3.2. Let's reiterate once more that the elements visualized by the square in Fig. 2(b) are not statements, but sets (more specifically: extensions of concepts). After all, the original Euler diagram in Fig. 2(a) also visualizes a relation (viz. proper inclusion) between two sets. Note, however, that this Euler diagram can also be seen as a visual representation of the categorical A-statement 'all horses are animals' ${ }^{11}$ One can then consider the corresponding I-, E- and O-statements, i.e. 'some horses are animals', 'no horses are animals' and 'some horses aren't animals'. Together, these four categorical statements yield another classical square of opposition. However, this second square is very different from the one shown in Fig. 2(b): the square in Fig. 2(b) is a diagram for sets and Aristotelian relations between sets (e.g. having empty or non-empty intersection), whereas the second square just described would be a diagram for statements and Aristotelian relations between statements (e.g. being able or not being able to be true together).

\footnotetext{
${ }^{11}$ Schopenhauer himself also took this view [28, p. 290].
} 
Figure 4. (a) Schopenhauer's Euler diagram; (b) the corresponding Aristotelian diagram: a degenerate square.

(a)

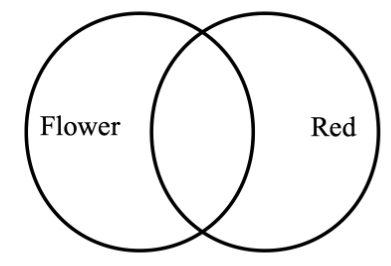

(b)

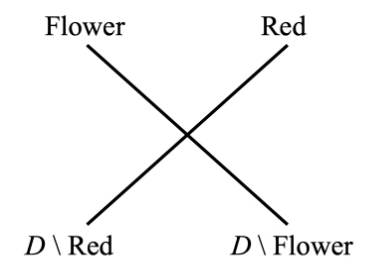

Remark 3.3. Recall that $\mathbb{B}_{3}$ is the smallest Boolean algebra of sets that nontrivially contains Horse and Animal. This non-triviality condition means that neither Horse nor Animal are identical to $D$ or $\emptyset$, i.e. to the top or bottom element of $\mathbb{B}_{3}$ (cf. the Hasse diagram in Fig. 3). Without this non-triviality condition, there is a smaller Boolean algebra of sets that contains Horse and Animal. This smaller Boolean algebra, $\mathbb{B}_{2}$, has $2^{2}=4$ elements; its top element is Animal and its bottom element is $\emptyset$; its two remaining elements are Horse and Animal $\backslash$ Horse. The Hasse diagram for $\mathbb{B}_{2}$ is shown in Fig. $3(\mathrm{~b})$; note that $\mathbb{B}_{2}$ is isomorphic to the powerset algebra $\wp(\{1,2\})$. The Boolean algebra of sets $\mathbb{B}_{2}$ contains Horse and Animal, but Animal ends up being identical to the top element of $\mathbb{B}_{2}$. Consequently, next to the 'expected' results, $\mathbb{B}_{2}$ also yields some very counter-intuitive results; for example, there is a $\mathbb{B}_{2}$-subalternation from Horse to Animal (because Horse $\subset$ Animal), but additionally, there is also a $\mathbb{B}_{2}$-subcontrariety between Horse and Animal (because Horse $\cap$ Animal $\neq \emptyset$ and Horse $\cup$ Animal $=$ Animal $)$. Furthermore, $\mathbb{B}_{2}$ does not contain enough elements to construct a square of opposition (recall that by definition, Aristotelian diagrams cannot contain a Boolean algebra's top or bottom elements). These issues illustrate the importance of respecting the non-triviality condition when transforming a given Euler diagram into an Aristotelian diagram.

\subsection{From an Euler Diagram to a Degenerate Square of Opposition}

Another of Schopenhauer's Euler diagrams illustrates the case where "Two spheres each include a part of the other." [29, p. 67]. The example he gives involves the concepts of Flower and Red; the accompanying Euler diagram is shown in Fig. 4(a). The smallest Boolean algebra of sets that non-trivially (recall Remark 3.3) contains Flower and Red has $2^{4}=16$ elements. This Boolean algebra, $\mathbb{B}_{4}$, has top element $D$ (for Domain), bottom element $\emptyset$, and four atomic elements, viz. Flower $\cap$ Red, Flower $\cap(D \backslash$ Red $),(D \backslash$ Flower $) \cap$ Red and $(D \backslash$ Flower $) \cap(D \backslash$ Red $)$. The Hasse diagram for $\mathbb{B}_{4}$ is shown in Fig. 5 ; note that $\mathbb{B}_{4}$ isomorphic to the powerset algebra $\wp(\{1,2,3,4\})$.

We can now determine the Aristotelian relations holding between some of the sets of this Boolean algebra $\mathbb{B}_{4}$ (again, recall Definition 2.3). First of all, since 
Figure 5. Hasse diagram for the Boolean algebra $\mathbb{B}_{4}$. Note that $\Delta$ denotes the symmetrical difference between two sets, i.e. $X \Delta Y:=(X \cap(D \backslash Y)) \cup((D \backslash X) \cap Y)$.

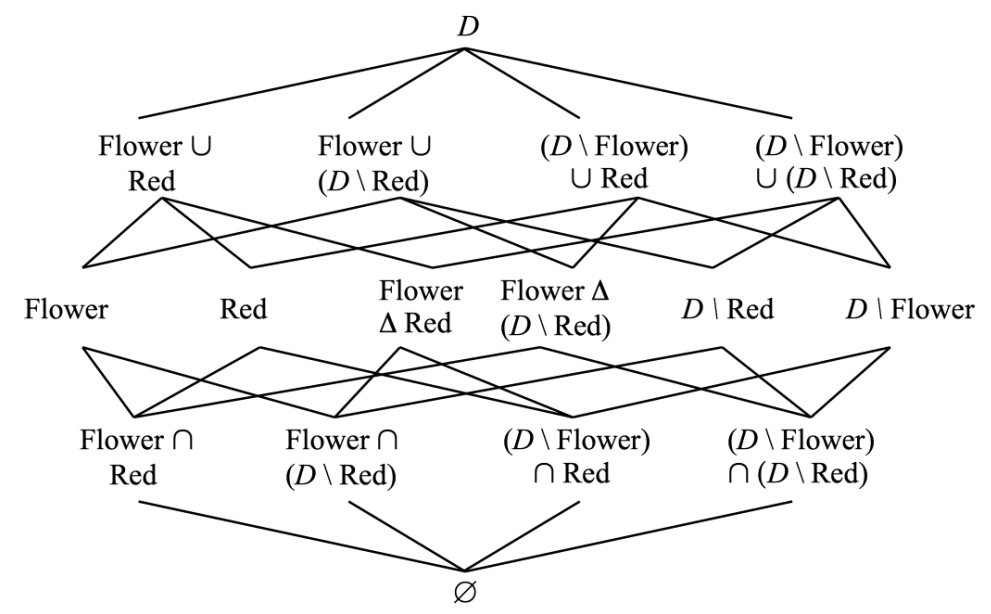

Flower $\cap(D \backslash$ Flower $)=\emptyset$ and Flower $\cup(D \backslash$ Flower $)=D$, it follows that Flower and $D \backslash$ Flower are $\mathbb{B}_{4}$-contradictories; similarly, Red and $D \backslash$ Red are also $\mathbb{B}_{4^{-}}$ contradictories. Furthermore, since (i) Flower $\cap$ Red $\neq \emptyset$, (ii) Flower $\cap(D \backslash$ Red $) \neq \emptyset$, (iii) $(D \backslash$ Flower $) \cap \operatorname{Red} \neq \emptyset$ and (iv) $(D \backslash$ Flower $) \cap(D \backslash$ Red $) \neq \emptyset$, it follows that Flower and Red are $\mathbb{B}_{4}$-unconnected, i.e. these two sets do not stand in any Aristotelian relation whatsoever in $\mathbb{B}_{4} \cdot{ }^{12}$ Completely analogously, one can show that Flower and $D \backslash$ Red are $\mathbb{B}_{4}$-unconnected, $D \backslash$ Flower and Red are $\mathbb{B}_{4}$-unconnected, and $D \backslash$ Flower and $D \backslash$ Red are $\mathbb{B}_{4}$-unconnected. This can all be summarized by means of a 'degenerate' square of opposition (or ' $\mathrm{X}$ of opposition' [4, pp. 11-12]), as shown in Fig. 4(b).

We have thus succeeded in transforming Schopenhauer's original Euler diagram (Fig. 4(a)) into an Aristotelian diagram, viz. a degenerate square of opposition (Fig. 4(b)). Once again, it bears emphasizing that the Aristotelian relations (or rather: lack thereof, in the four cases of unconnectedness) that are visualized

\footnotetext{
${ }^{12}$ The Euler diagram in Fig. 4(a) can be seen as a visual representation of the statement 'some flowers are red', as acknowledged by Schopenhauer [28, p. 294]. This means exactly that Flower $\cap$ Red $\neq \emptyset$; cf. condition (i) above. However, Schopenhauer explicitly indicates that this same Euler diagram also represents the statements 'some flowers are not red' and 'some red things are not flowers' [28, p. 294], i.e. Flower $\cap(D \backslash$ Red $) \neq \emptyset$ and $(D \backslash$ Flower $) \cap$ Red $\neq \emptyset$; cf. conditions (ii) and (iii) above. As far as I know, Schopenhauer never explicitly discussed the interpretation 'some non-red things are not flowers' of this same Euler diagram, which corresponds to condition (iv) above. Finally, note that by attaching equal importance to the four regions Flower $\cap$ Red, Flower $\cap(D \backslash$ Red $),(D \backslash$ Flower $) \cap$ Red and ( $D \backslash$ Flower $) \cap(D \backslash$ Red), we are essentially reinterpreting the diagram in Fig. 4(a) as a Venn diagram.
} 
by this square are all mathematically well-defined: they hold relative to the underlying Boolean algebra $\mathbb{B}_{4}$ (cf. Fig. 5), and are thus specific instantiations of the general characterizations of the Aristotelian relations and unconnectedness provided by Definitions 2.1 and 2.2.

Remarks $3.1-3.3$ from the previous subsection continue to apply in the present situation. For example, we once again observe the non-injective nature of the transformation process from Euler diagrams to Aristotelian diagrams. In particular, note that the Euler diagram in Fig. 4(a), which visualizes that Flower $\cap$ Red $\neq \emptyset$, has been transformed into the degenerate square of opposition in Fig. 4(b), but that another Euler diagram, for example one which visualizes that $(D \backslash$ Flower $) \cap$ $(D \backslash$ Red $) \neq \emptyset$, would be transformed into exactly the same degenerate square. Alternatively, one can again view the single Euler diagram in Fig. 4(a) as a visual representation of six relations (or rather: lack thereof, in the four cases of unconnectedness) among Flower, Red, $D \backslash$ Flower and $D \backslash$ Red, all six of which are also visualized by the degenerate square in Fig. 4(b). Also note that the Euler diagram in Fig. 4(a) can be viewed as a visual representation of the categorical I-statement 'some flowers are red' (cf. Footnote 12). One can then consider the corresponding A-, E- and O-statements, i.e. 'all flowers are red', 'no flowers are red' and 'some flowers are not red'. Together, these four categorical statements yield a classical square of opposition. The difference between these two squares is now even clearer than in Subsection 3.1: the diagram in Fig. 4(b) is a degenerate square of opposition for sets, whereas the second diagram just described would be a classical square for statements.

To conclude this section, let's summarize the results that have been obtained. It is well-known in contemporary logical geometry that there are exactly two types of Aristotelian squares, viz. classical squares and degenerate squares. In Subsection 3.1 we have seen that one of Schopenhauer's Euler diagrams can be transformed into a classical square, and in Subsection 3.2 we have seen that another one of his diagrams can be transformed into a degenerate square. In other words, Schopenhauer's stock of Euler diagrams is sufficiently rich so as to contain analogues of each of the two types of Aristotelian squares that are nowadays studied in logical geometry.

\section{Partitions, Euler Diagrams and Aristotelian Diagrams}

In this section we will deal with yet another Euler diagram that was used by Schopenhauer — or rather, an entire class of Euler diagrams. ${ }^{13}$ These diagrams illustrate the case where "A sphere includes two or more further spheres, which are mutually exclusive and at the same time exhaust the first sphere" [29, p. 66].

\footnotetext{
${ }^{13}$ Strictly speaking, it might be better to call the diagrams used by Schopenhauer that are discussed in this section (and the next one) 'Eulerian diagrams' or 'Euler-type diagrams', rather than simply 'Euler diagrams', because they do not have direct counterparts in Euler's original writings.
} 
Figure 6. (a) Schopenhauer's Euler diagram; (b) the corresponding Aristotelian diagram: a strong JSB hexagon.

(a)

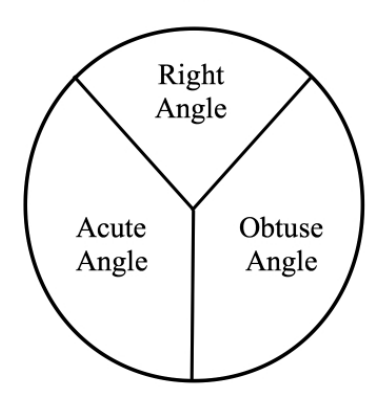

(b)

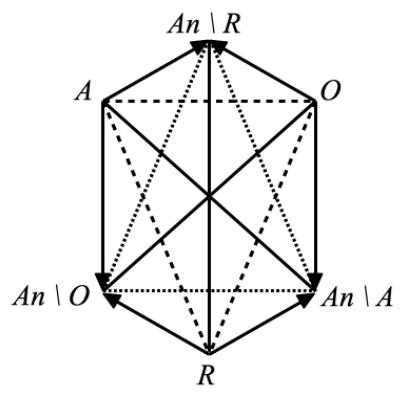

The example he gives involves the concepts of Acute Angle, Right Angle and Obtuse Angle; the accompanying Euler diagram is shown in Fig. 6(a). The smallest Boolean algebra of sets that non-trivially contains Acute Angle, Right Angle and Obtuse Angle has $2^{3}=8$ elements. This Boolean algebra, which we will again label $\mathbb{B}_{3}$, has top element $A n$ (for Angle), ${ }^{14}$ bottom element $\emptyset$, and three atomic elements, viz. $A$ (for Acute Angle), $R$ (for Right Angle) and $O$ (for Obtuse Angle).

We can now determine the Aristotelian relations holding between some of the sets of this Boolean algebra $\mathbb{B}_{3}$ (recall Definition 2.3). Since Acute Angle, Right Angle and Obtuse Angle are mutually exclusive, we have $A \cap R=\emptyset, A \cap O=\emptyset$ and $R \cap O=\emptyset$. Furthermore, since neither of these three concepts' extensions are empty, it follows that resp. $R \cup O \neq A n, A \cup O \neq A n$ and $A \cup R \neq A n$. We thus find that there are pairwise $\mathbb{B}_{3}$-contrarieties between each of $A, R$ and $O$. These three sets thus constitute a triangle of contraries in $\mathbb{B}_{3}$. Completely analogously, one can show that, for all distinct $X, Y \in\{A, R, O\}$, there are $\mathbb{B}_{3}$-contradictions between $X$ and $A n \backslash X$, as well as $\mathbb{B}_{3}$-subcontrarieties between $A n \backslash X$ and $A n \backslash Y$ and $\mathbb{B}_{3}$-subalternations from $X$ to $A n \backslash Y$. All these Aristotelian relations can be summarized by means of a JSB hexagon, as shown in Fig. 6(b).

Schopenhauer's remark that Acute Angle, Right Angle and Obtuse Angle are mutually exclusive thus essentially means that his Euler diagram for these three concepts can be transformed into a well-defined Aristotelian diagram, viz. a JSB

\footnotetext{
${ }^{14}$ Since Acute Angle, Right Angle and Obtuse Angle are meant to "exhaust the first sphere", this first sphere has to be interpreted as a 'restricted domain of discourse', i.e. Angle. Of course, there also exist objects that are not angles (e.g. flowers and horses), but if these were also taken into account, then Acute Angle, Right Angle and Obtuse Angle would not exhaust the domain of discourse.
} 
hexagon. However, Schopenhauer also notes that these three spheres are jointly exhaustive, i.e. $A \cup R \cup O=A n$. This information goes beyond the Aristotelian relations ${ }^{15}$ and provides additional Boolean information. This additional information determines the Boolean subtype of the Aristotelian diagram under consideration: the hexagon in Fig. 6(b) is a strong JSB hexagon.

The Euler diagram in Fig. 6(a) has thus been transformed into the strong JSB hexagon in Fig. 6(b). This should not come as a surprise. After all, Schopenhauer's remarks that Acute Angle, Right Angle and Obtuse Angle are mutually exclusive and jointly exhaustive mean that the set $\Pi:=\{A, R, O\}$ is a 3-partition of $\mathbb{B}_{3}$. By Theorem 2.7 (item 2), this 3-partition gives rise to a strong $\alpha_{3}$-structure, i.e. a strong JSB hexagon, which has the three elements of $\Pi$ on its triangle of contraries. This JSB hexagon is exactly the diagram shown in Fig. 6(b).

Although the example involving Acute/Right/Obtuse Angle is the only one that is explicitly given by Schopenhauer in [29], his theoretical discussion is much more general than this. Recall his description: "A sphere includes two or more further spheres, which are mutually exclusive and at the same time exhaust the first sphere" [29, p. 66, my emphasis]. The number of (mutually exclusive and jointly exhaustive) spheres that are included in the first sphere is thus left unspecified. The concrete Acute/Right/Obtuse Angle example is based on three spheres, but Schopenhauer could equally easily have given examples based on two spheres, four spheres, etc.

In his university lecture notes [28, p. 296], Schopenhauer explicitly provides an example of a sphere that includes two further spheres which are mutually exclusive and jointly exhaustive. His example involves the concepts of Organic and Inorganic (both included in Body [Körper]); the accompanying Euler diagram is shown in Fig. 7(a). The smallest Boolean algebra that non-trivially contains Organic and Inorganic has 4 elements. This Boolean algebra, which we will again label $\mathbb{B}_{2}$, has top element Body, bottom element $\emptyset$, and two atomic elements, viz. Organic and Anorganic. The Hasse diagram for $\mathbb{B}_{2}$ is shown in Fig. $7(\mathrm{~b})$. Since Organic $\cap$ Anorgnic $=\emptyset$ and Organic $\cup$ Anorganic $=$ Body, it follows that Organic and Anorganic are $\mathbb{B}_{2}$-contradictory. Schopenhauer's Euler diagram in Fig. 7 (a) can thus be transformed into the (very simple) Aristotelian diagram in Fig. 7(c). This Aristotelian diagram is a PCD. Again, this should not come as a surprise. After all, Schopenhauer's remarks that Organic and Anorganic are mutually exclusive and jointly exhaustive mean that the set \{Organic, Anorganic $\}$ is a 2-partition of $\mathbb{B}_{2}$. By Theorem 2.7 (item 1), this 2-partition gives rise to an $\alpha_{1}$-structure, i.e. a PCD. This PCD is exactly the diagram shown in Fig. $7(\mathrm{c})$.

As far as I know, Schopenhauer nowhere discussed an example of a sphere that includes four further spheres which are mutually exclusive and jointly exhaustive. However, one can easily construct such an example, cf. the Euler diagram shown in Fig. 8(a). The smallest Boolean algebra of sets that contains $A, B, C$ and $D$

\footnotetext{
${ }^{15}$ Again, note that the Aristotelian relations are binary in nature, and thus cannot capture the information that $A \cup R \cup O=A n$.
} 
Figure 7. (a) Schopenhauer's Euler diagram; (b) Hasse diagram for the Boolean algebra $\mathbb{B}_{2} ;(c)$ the corresponding Aristotelian diagram: a PCD.

(a)

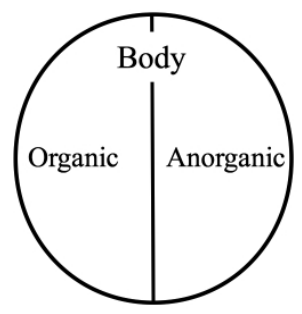

(b)

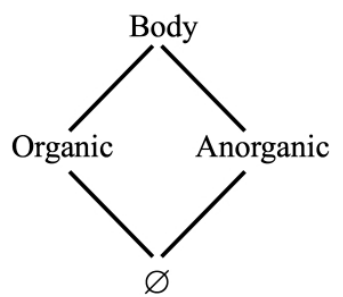

(c)

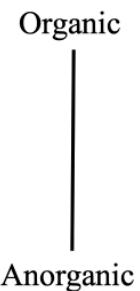

FiguRE 8. (a) Schopenhauer-inspired Euler diagram; (b) the corresponding Aristotelian diagram: a strong Moretti octagon.

(a)

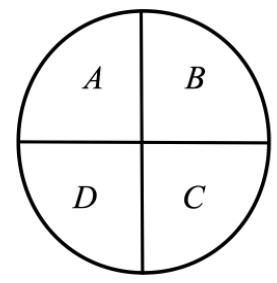

(b)

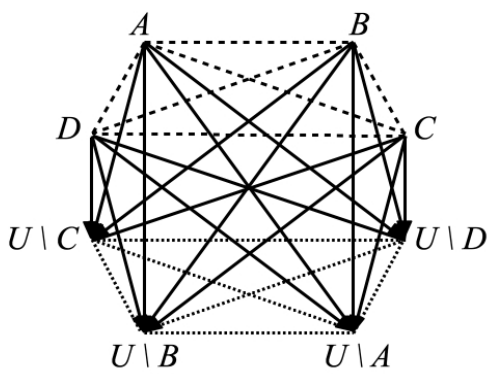

will again be called $\mathbb{B}_{4}$. This Boolean algebra has 16 elements in total, including top element $U$ (for Universe), bottom element $\emptyset$, and four atomic elements, viz. $A$, $B, C$ and $D$. One can easily determine the Aristotelian relations that hold among $A, B, C, D$ and their complements in $\mathbb{B}_{4}$. This yields a Moretti octagon, as shown in Fig. 8(b). Furthermore, since $A, B, C$ and $D$ are jointly exhaustive (i.e. $A \cup B \cup$ $C \cup D=U)$, the diagram in Fig. 8(b) is a strong Moretti octagon. Once again, this should not come as a surprise. After all, the fact that $A, B, C$ and $D$ are mutually exclusive and jointly exhaustive means that the set $\Pi:=\{A, B, C, D\}$ is a 4-partition of $\mathbb{B}_{4}$. By Theorem 2.7 (item 2), this 4-partition gives rise to a strong $\alpha_{4}$-structure, i.e. a strong Moretti octagon, which has the four elements of $\Pi$ on its trapezoid of contraries. This strong Moretti octagon is exactly the diagram shown in Fig. 8(b).

I will again finish this section by summarizing the results that have been obtained. It is well-known in contemporary logical geometry that there is a precise 
correspondence between partitions and $\alpha$-structures (cf. Theorem 2.7). We have seen that Schopenhauer explicitly discussed Euler diagrams where a given sphere contains two or more spheres that are mutually exclusive and jointly exhaustive - i.e. that constitute a partition of the first sphere. His concrete example of a 2-partition [28, p. 296] can be transformed into a PCD, i.e. an $\alpha_{1}$-structure, which is in line with item 1 of Theorem 2.7. His concrete example of a 3-partition [29, p. 66] can be transformed into a strong JSB hexagon, i.e. a strong $\alpha_{3}$-structure, which is in line with item 2 of Theorem 2.7. For the sake of illustration, I have also discussed an example of a 4-partition, showing that it can be transformed into a strong Moretti octagon, i.e. a strong $\alpha_{4}$-structure, which is again in line with item 2 of Theorem 2.7. Furthermore, since Schopenhauer left the number of mutually exclusive and jointly exhaustive spheres that are included in the first sphere unspecified, he was implicitly considering an infinite series of Euler diagrams (for each $n \geq 2$, there is a distinct Euler diagram corresponding to an $n$-partition). Each Euler diagram in this series can be transformed into a distinct Aristotelian diagram, viz. an $\alpha$-structure (from $n=3$ onwards, this $\alpha_{n}$-structure is a strong one). In other words, by leaving the number of cells in the partition unspecified, Schopenhauer essentially anticipated the entire infinite series of $\alpha$-structures from contemporary logical geometry.

\section{Boolean Subtypes of Aristotelian Diagrams}

In this section we consider a final class of Euler diagrams discussed by Schopenhauer. These diagrams illustrate the case where "Two spheres lie inside a third, but do not exhaust it" [29, p. 67]. The example he gives involves the concepts of Water and Earth, both of which lie inside Matter. The accompanying Euler diagram is shown in Fig. 9(a). The smallest Boolean algebra of sets that non-trivially contains Water and Earth has $2^{3}=8$ elements. This Boolean algebra, which we will again label $\mathbb{B}_{3}$, has top element $M$ (for Matter), bottom element $\emptyset$, and three atomic elements, viz. $W$ (for Water), $E$ (for Earth) and $M \backslash(W \cup E) .{ }^{16}$

We can now determine the Aristotelian relations holding between some of the sets of this Boolean algebra $\mathbb{B}_{3}$ (recall Definition 2.3). Since Water and Earth are mutually exclusive, we have $W \cap E=\emptyset .{ }^{17}$ Furthermore, since Water and Earth do not exhaust Matter, we have $W \cup E \neq M$. This means that $W$ and $E$ are $\mathbb{B}_{3^{-}}$ contrary. Completely analogously, one can show that there are $\mathbb{B}_{3}$-contradictions between $W$ and $M \backslash W$ and between $E$ and $M \backslash E$, as well as $\mathbb{B}_{3}$-subalternations from $W$ to $M \backslash E$ and from $E$ to $M \backslash W$, and finally, a $\mathbb{B}_{3}$-subcontrariety between $M \backslash E$ and $M \backslash W$. All these Aristotelian relations can be summarized by means of a classical square of opposition, as shown in Fig. 9(b).

\footnotetext{
${ }^{16}$ Since Water and Earth do not exhaust matter, it follows that $M \backslash(W \cup E) \neq \emptyset$.

${ }^{17}$ Schopenhauer does not explicitly say that the two spheres that lie inside the third one have to be mutually exclusive, but his Euler diagram does display them as such; cf. Fig. 9(a). Furthermore, immediately after giving the example, he does state that "The last case applies to all concepts whose spheres do not have anything directly in common" [29, p. 67, my emphasis].
} 
Figure 9. (a) Schopenhauer's Euler diagram; (b) first corresponding Aristotelian diagram: a classical square of opposition; (c) second corresponding Aristotelian diagram: a strong JSB hexagon.

(a)

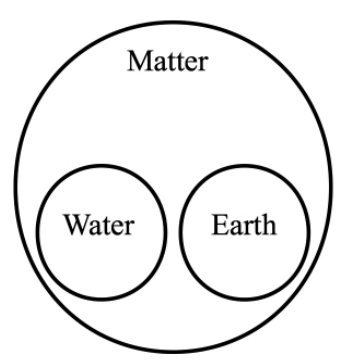

(b)

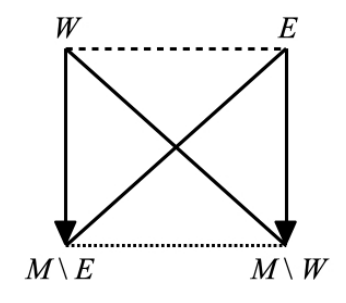

(c)

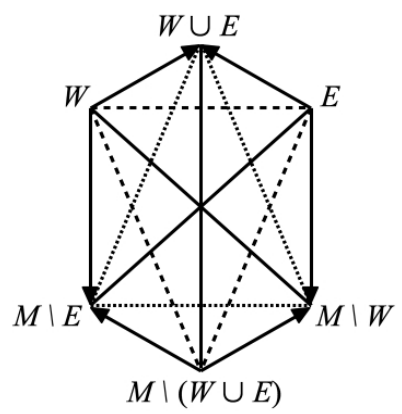

The Euler diagram in Fig. 9(a) has thus been transformed into a well-defined Aristotelian diagram, viz. the classical square of opposition in Fig. 9(b). However, if we consider not only $W$ and $E$ (and their complements), but also the third atomic element of $\mathbb{B}_{3}$, i.e. $M \backslash(W \cup E)$ (and its complement), then this very same Euler diagram can also be transformed into another Aristotelian diagram. One can easily show that $M \backslash(W \cup E)$ is $\mathbb{B}_{3}$-contrary to $W$ as well as to $E$. These three sets thus constitute a triangle of contraries in $\mathbb{B}_{3}$. By also taking the three other non-trivial elements of $\mathbb{B}_{3}$ into account, we can construct a JSB hexagon, as shown in Fig. 9(c). Although Water and Earth by themselves do not exhaust Matter (i.e. $W \cup E \neq M)$, adding this third 'remainder' concept $M \backslash(W \cup E)$ does exhaust Matter (i.e. $W \cup E \cup M \backslash(W \cup E)=M)$ ). This means that the diagram in Fig. 9(c) is a strong JSB hexagon. This should not come as a surprise. After all, Schopenhauer's remarks that Water and Earth are mutually exclusive, together with the definition of $M \backslash(W \cup E)$ as a 'remainder' concept, imply that the set $\Pi:=\{W, E, M \backslash(W \cup E)\}$ is a 3-partition of $\mathbb{B}_{3}$. By Theorem 2.7 (item 2), this 3 -partition gives rise to a strong $\alpha_{3}$-structure, i.e. a strong JSB hexagon, which has the three elements of $\Pi$ on its triangle of contraries. This JSB hexagon is exactly the diagram shown in Fig. 9(c).

The Euler diagram in Fig. 9(a) can thus be transformed into a classical square, i.e. an $\alpha_{2}$-structure, but also into a strong JSB hexagon, i.e. a strong $\alpha_{3^{-}}$ structure. An analogous situation arises when we move to Euler diagrams with higher numbers of spheres. Schopenhauer himself only considered and illustrated the case where "Two [disjoint] spheres lie inside a third, but do not exhaust it" [29, p. 67, my emphasis], and unlike the case discussed in Section 4, he did not generalize this to higher numbers of spheres. I will now discuss two such generalizations, which are completely in line with Schopenhauer's thinking. 
Figure 10. (a) Schopenhauer-inspired Euler diagram; (b) first corresponding Aristotelian diagram: a weak JSB hexagon; (c) second corresponding Aristotelian diagram: a strong Moretti oc-

tagon.

(a)

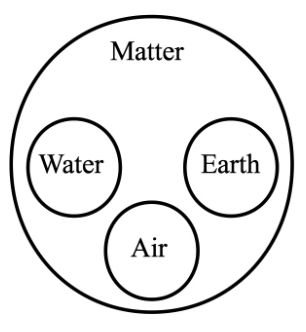

(b)

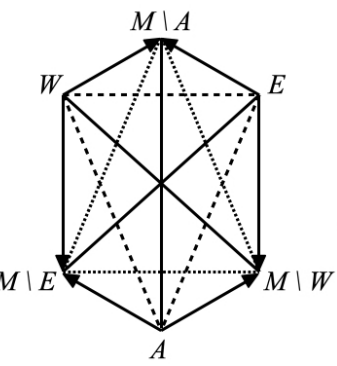

(c)

The first generalization can be described as a case where "three disjoint spheres lie inside a fourth, but do not exhaust it". A concrete example involves the concepts of Water, Earth and Air, all of which lie inside Matter; cf. the Euler diagram in Fig. 10(a). The smallest Boolean algebra of sets that non-trivially contains Water, Earth and Air has $2^{4}=16$ elements. This Boolean algebra, which we will again label $\mathbb{B}_{4}$, has top element $M$ (for Matter), bottom element $\emptyset$, and four atomic elements, viz. $W$ (for Water), $E$ (for Earth), $A$ (for Air) and $M \backslash(W \cup E \cup A)$. One can easily determine the Aristotelian relations that hold among Water, Earth and Air and their complements in $\mathbb{B}_{4}$. This yields a JSB hexagon, as shown in Fig. 10(b). Since Water, Earth and Air do not exhaust Matter, this diagram is a weak JSB hexagon, i.e. a weak $\alpha_{3}$-structure. If we consider not only $W$, $E$ and $A$ (and their complements), but also the fourth atomic element of $\mathbb{B}_{4}$, i.e. $M \backslash(W \cup E \cup A)$ (and its complement), then the Euler diagram in Fig. 10(a) can also be transformed into another Aristotelian diagram. One can easily show that $M \backslash(W \cup E \cup A)$ is $\mathbb{B}_{4}$-contrary to $W$, to $E$ and to $A$. These four sets thus constitute a trapezoid of contraries in $\mathbb{B}_{4}$. By also taking the four complements of $W, E, A$ and $M \backslash(W \cup E \cup A)$ into account, we can construct a Moretti octagon, as shown in Fig. 10(c). Although Water, Earth and Air by themselves do not exhaust Matter (i.e. $W \cup E \cup A \neq M$ ), adding this fourth 'remainder' concept $M \backslash(W \cup E \cup A)$ does exhaust Matter (i.e. $W \cup E \cup A \cup M \backslash(W \cup E \cup A)=M$ ). This means that the diagram in Fig. 10(c) is a strong Moretti octagon. Once again, this should not come as a surprise. After all, the fact that Water, Earth are Air are mutually exclusive, together with the definition of $M \backslash(W \cup E \cup A)$ as a 'remainder' concept, implies that the set $\Pi:=\{W, E, A, M \backslash(W \cup E \cup A)\}$ is a 4-partition of $\mathbb{B}_{4}$. By Theorem 2.7 (item 2), this 4-partition gives rise to a strong $\alpha_{4}$-structure, i.e. a strong Moretti octagon, which has the four elements of $\Pi$ on its trapezoid of contraries. This Moretti octagon is exactly the diagram shown in Fig. 10(c). 
FiguRE 11. (a) Schopenhauer-inspired Euler diagram; (b) one corresponding Aristotelian diagram: a weak Moretti octagon. (There also exists another one, which is not shown here.)

(a)

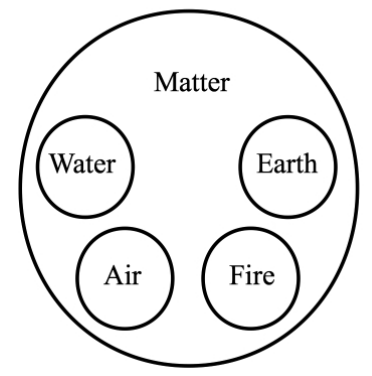

(b)

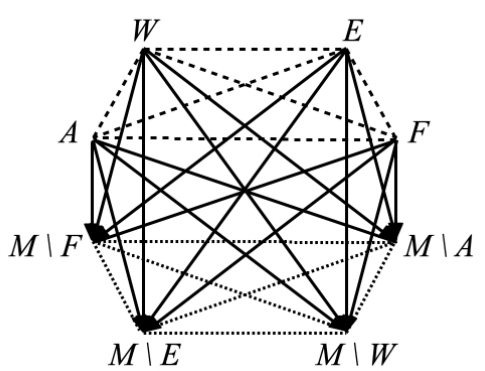

I now briefly turn to a second generalization, which can be described as a case where "four disjoint spheres lie inside a fifth, but do not exhaust it". A concrete example involves the concepts of Water, Earth, Air and Fire, all of which lie inside Matter; cf. the Euler diagram in Fig. 11(a). The smallest Boolean algebra of sets that non-trivially contains Water, Earth, Air and Fire has $2^{5}=32$ elements. This Boolean algebra, $\mathbb{B}_{5}$, has top element $M$ (for Matter), bottom element $\emptyset$, and five atomic elements, viz. $W$ (for Water), $E$ (for Earth), $A$ (for Air), $F$ (for Fire) and $M \backslash(W \cup E \cup A \cup F)$. One can easily show that Water, Earth, Air and Fire yield a Moretti octagon in $\mathbb{B}_{5}$, as shown in Fig. 11(b). Since Water, Earth, Air and Fire do not exhaust Matter, this diagram is a weak Moretti octagon, i.e. a weak $\alpha_{4}$ structure. If we consider not only $W, E, A$ and $F$ (and their complements), but also the fifth atomic element of $\mathbb{B}_{5}$, i.e. $M \backslash(W \cup E \cup A \cup F)$ (and its complement), then the Euler diagram in Fig. 11(a) can also be transformed into another Aristotelian diagram, viz. an $\alpha_{5}$-structure (which is not shown here, for reasons of space). Although Water, Earth, Air and Fire by themselves do not exhaust Matter (i.e. $W \cup$ $E \cup A \cup F \neq M)$, adding this fifth 'remainder' concept $M \backslash(W \cup E \cup A \cup F)$ does exhaust Matter (i.e. $W \cup E \cup A \cup F \cup M \backslash(W \cup E \cup A \cup F)=M)$. This means that the $\alpha_{5}$-structure is a strong $\alpha_{5}$-structure, which is again perfectly in line with item 2 of Theorem 2.7, since $\{W, E, A, F, M \backslash(W \cup E \cup A \cup F)\}$ is a 5-partition of $\mathbb{B}_{5}$.

By now, the pattern that emerges should be very clear:

- The Euler diagram in Fig. 9(a) can be transformed into an $\alpha_{2}$-structure (Fig. 9(b)), but also into a strong $\alpha_{3}$-structure (Fig. 9(c)). ${ }^{18}$

- The Euler diagram in Fig. 10(a) can be transformed into a weak $\alpha_{3}$-structure (Fig. 10(b)), but also into a strong $\alpha_{4}$-structure (Fig. 10(c)).

\footnotetext{
${ }^{18}$ Since the family of classical squares is Boolean homogeneous, it makes little sense to talk about a 'weak' $\alpha_{2}$-structure (recall Theorem 2.6, item 2).
} 
- The Euler diagram in Fig. 11(a) can be transformed into a weak $\alpha_{4}$-structure (Fig. 11(b)), but also into a strong $\alpha_{5}$-structure (not shown).

We can now combine these results with those of Section 4 . In that section, we have seen that whenever $n \geq 3$ mutually disjoint spheres lie inside a given sphere and also exhaust that sphere, they give rise to a strong $\alpha_{n}$-structure. In this section, we have seen that whenever $n \geq 3$ mutually disjoint spheres lie inside a given sphere but do not exhaust it, they give rise to a weak $\alpha_{n}$-structure. ${ }^{19}$. By explicitly distinguishing between cases where $n \geq 3$ mutually disjoint spheres are jointly exhaustive and cases where they are not jointly exhaustive, Schopenhauer thus clearly anticipated the distinction between strong and weak $\alpha_{n}$-structures (cf. Theorem 2.6), and hence, more generally, the insight that families of Aristotelian diagrams can have distinct Boolean subtypes.

\section{Conclusion}

In this paper I have explored the connection between Schopenhauer's Euler diagrams and the Aristotelian diagrams that are studied in contemporary logical geometry. One can define the Aristotelian relations in a very general fashion (relative to arbitrary Boolean algebras), which allows us to define not only Aristotelian diagrams for statements, but also for sets. I have shown that, once this generalization has been made, each of Schopenhauer's concrete Euler diagrams can be transformed into a well-defined Aristotelian diagram. More importantly, I have also argued that Schopenhauer had several more general, systematic insights about Euler diagrams, which anticipate general insights and theorems about Aristotelian diagrams in contemporary logical geometry.

For example, it is well-known in logical geometry today that there are exactly two types of Aristotelian squares, viz. classical squares and degenerate squares. Schopenhauer had Euler diagrams that can be transformed into each of these two types of Aristotelian squares (cf. Section 3). Furthermore, logical geometry shows that there is a clear correspondence between $n$-partitions and (strong) $\alpha_{n^{-}}$ structures. Schopenhauer anticipated this correspondence, by considering Euler diagrams for $n$-partitions, each of which can be transformed into the corresponding (strong) $\alpha_{n}$-structure; he also discussed this correspondence in its full generality, i.e. by considering $n$-partitions for arbitrary $n$ (cf. Section 4 ). Finally, logical geometry emphasizes that many families of Aristotelian diagrams have distinct Boolean subtypes. In particular, for $n \geq 3$, the family of $\alpha_{n}$-structures has two Boolean subtypes (strong and weak). By explicitly distinguishing between cases where a number of (mutually disjoint) spheres are jointly exhaustive and cases where they are not jointly exhaustive, Schopenhauer also displayed a remarkable sensitivity to the subtle interplay between Aristotelian and Boolean considerations (cf. Section 5).

\footnotetext{
$\overline{{ }^{19} \text { Recall that for }} n<3$, the family of $\alpha_{n}$-structures is Boolean homogeneous; cf. items 1 and 2 of Theorem 2.6.
} 
In sum: because of his various concrete Euler diagrams and, especially, his more systematic observations about them, Schopenhauer can rightly be considered a distant forerunner of contemporary logical geometry, which studies Aristotelian diagrams as objects of independent mathematical and philosophical interest.

\section{Acknowledgments}

I would like to thank Jens Lemanski, Hans Smessaert and Margaux Smets for their comments on earlier versions of this paper. The research reported in this paper is financially supported through a Postdoctoral Fellowship from the Research Foundation-Flanders (FWO).

\section{References}

[1] I. Anellis, The Genesis of the Truth-Table Device. Russell: the Journal of Bertrand Russell Studies 24 (2004), 55 - 70.

[2] M. E. Baron, A Note on the Historical Development of Logic Diagrams. Leibniz, Euler and Venn. The Mathematical Gazette 53 (1969), 113 - 125.

[3] P. Bernhard, Euler-Diagramme: Zur Morphologie einer Repräsentationsform in der Logik. Mentis, 2001.

[4] J.-Y. Béziau and G. Payette, Preface. In J.-Y. Béziau and G. Payette (eds.), The Square of Opposition. A General Framework for Cognition, Peter Lang (2012), 9 22 .

[5] R. Blanché, Structures Intellectuelles. Essai sur l'Organisation Systématique des Concepts. Vrin, 1969.

[6] L. Demey, Using Syllogistics to Teach Metalogic. Metaphilosophy 48 (2017), 575 590.

[7] L. Demey, Computing the Maximal Boolean Complexity of Families of Aristotelian Diagrams. Journal of Logic and Computation 28 (2018), 1323 - 1339.

[8] L. Demey, Aristotelian Diagrams for Semantic and Syntactic Consequence. Synthese (forthcoming).

[9] L. Demey, Boolean Considerations on John Buridan's Octagons of Opposition. History and Philosophy of Logic (forthcoming).

[10] L. Demey, Metalogic, Metalanguage and Logical Geometry. Logique et Analyse (forthcoming).

[11] L. Demey and H. Smessaert, The Relationship between Aristotelian and Hasse Diagrams. In T. Dwyer, H. Purchase and A. Delaney (eds.), Diagrammatic Representation and Inference, Lecture Notes in Computer Science 8578, Springer (2014), 213 - 227.

[12] L. Demey and H. Smessaert, Metalogical Decorations of Logical Diagrams. Logica Universalis 10 (2016), 233 - 292.

[13] L. Demey and H. Smessaert, The Interaction between Logic and Geometry in Aristotelian Diagrams. In M. Jamnik, Y. Uesaka and S. Elzer Schwartz (eds.), Diagrammatic Representation and Inference, Lecture Notes in Computer Science 9781, Springer (2016), $67-82$. 
[14] L. Demey and H. Smessaert, Aristotelian and Duality Relations beyond the Square of Opposition. In P. Chapman, G. Stapleton, A. Moktefi, S. Perez-Kriz and F. Bellucci (eds.), Diagrammatic Representation and Inference, Lecture Notes in Computer Science 10871, Springer (2018), $640-656$.

[15] L. Demey and H. Smessaert, Combinatorial Bitstring Semantics for Arbitrary Logical Fragments. Journal of Philosophical Logic 47 (2018), 325 - 363.

[16] L. Demey and H. Smessaert, Geometric and Cognitive Differences between Aristotelian Diagrams for the Boolean Algebra $\mathbb{B}_{4}$. Annals of Mathematics and Artificial Intelligence $\mathbf{8 3}$ (2018), 185 - 208.

[17] S. Givant and P. Halmos, Introduction to Boolean Algebras. Springer, 2009.

[18] P. Jacoby, A Triangle of Opposites for Types of Propositions in Aristotelian Logic. The New Scholasticism 24 (1950), 32 - 56.

[19] J. N. Keynes, Studies and Exercises in Formal Logic. Fourth Edition. MacMillan, 1906.

[20] C. J. W. Kloesel (ed.), Writings of Charles Sanders Peirce. A Chronological Edition. Volume 4: 1879 - 1884. Indiana University Press, 1989.

[21] J. Lemanski, Means or End? On the Valuation of Logic Diagrams. Logiko-Filosofskie Studii 14 (2016), 98 - 121.

[22] J. Lemanski, Periods in the Use of Euler-Type Diagrams. Acta Baltica Historiae et Philosophiae Scientiarum 5 (2017), 50 - 69.

[23] J. Lemanski, Logic Diagrams in the Weigel and Weise Circles. History and Philosophy of Logic 39 (2018), 3 - 28.

[24] J. Lemanski and A. Moktefi, Making Sense of Schopenhauer's Diagram of Good and Evil. In P. Chapman, G. Stapleton, A. Moktefi, S. Perez-Kriz and F. Bellucci (eds.), Diagrammatic Representation and Inference, Lecture Notes in Computer Science 10871, Springer (2018), $721-724$.

[25] A. Moretti, The Geometry of Logical Opposition. PhD thesis, University of Neuchâtel, 2009.

[26] R. Pellissier, "Setting" n-Opposition. Logica Universalis 2 (2008), 235 - 263.

[27] H. Reichenbach, The Syllogism Revised. Philosophy of Science 19 (1952), 1 - 16.

[28] A. Schopenhauer, Theorie des gesammten Vorstellens, Denkens und Erkennens. Philosophische Vorlesungen, Teil I (edited by V. Spierling). Piper, 1986.

[29] A. Schopenhauer, The World as Will and Representation, Volume 1 (translated and edited by J. Norman, A. Welchman and C. Janaway). Cambridge University Press, 2010.

[30] A. Sesmat, Logique II. Les Raisonnements. Hermann, 1951.

[31] H. Smessaert and L. Demey, Logical Geometries and Information in the Square of Opposition. Journal of Logic, Language and Information 23 (2014), 527 - 565.

[32] H. Smessaert and L. Demey, Logical and Geometrical Complementarities between Aristotelian Diagrams. In T. Dwyer, H. Purchase and A. Delaney (eds.), Diagrammatic Representation and Inference, Lecture Notes in Computer Science 8578, Springer (2014), $246-260$. 
[33] H. Smessaert and L. Demey, Béziau's Contributions to the Logical Geometry of Modalities and Quantifiers. In A. Koslow and A. Buchsbaum (eds.), The Road to Universal Logic, Volume I, Springer (2015), 475 - 494.

[34] H. Smessaert and L. Demey, The Unreasonable Effectiveness of Bitstrings in Logical Geometry. In J.-Y. Béziau and G. Basti (eds.), The Square of Opposition: A Cornerstone of Thought, Springer (2017), $197-214$.

[35] R. Wicks, Arthur Schopenhauer. In E. Zalta (ed.), Stanford Encyclopedia of Philosophy (Summer 2017 Edition), CSLI (2017).

Lorenz Demey

Center for Logic and Philosophy of Science

KU Leuven

Kardinaal Mercierplein 2

3000 Leuven, Belgium

e-mail: lorenz.demey@kuleuven.be 